IRA International Journal of Management \& Social Sciences ISSN 2455-2267

Vol. 14, Issue 02 (Special Issue) pg. 94-100.

International Conference on Wellbeing: Lifespan Perspectives E̊ Practices for Sustainable

Communities, 2019.

\title{
Our Health through Integrated Process - A Review
}

Aparna Sharma

Anantham Mind Studio, New Delhi, India.

DOI: $10.21013 /$ jmss.v14.n2sp.p12

(C) Authors.

Type of Review: Peer Reviewed under the Responsibility of the conference's Scientific Committee.

Disclaimer: The copyright to this work is retained by the authorship. This work contains the opinions ${ }^{\circ}$ views of the authorship solely and the same are not the views or opinions of the IRA. IRA disclaims of any harm or loss caused due to the published content to any party. 


\begin{abstract}
Working on integrating olden, traditional ways of alternative medicine, yogic techniques and positive psychology with mindfulness meditations, the author felt the alarming rates of depression is increasing in the society, intolerance, impatience and stress being the most evidently seen which led to this approach of positive psychology integrating with some alternative modalities. Many chronic disorders, diseases are largely out of severe stress which leads to psychosomatic illness and disorders. This present paper attempts to review the relation between psychosomatic illness and alternative medicine impact in treating patients. Also suggest further to use this as remedial practice for the future.
\end{abstract}

Keywords: Holistic and Alternate Medicine, Mental Health, Wellbeing.

\title{
Introduction
}

With excess digital exposure, we are overloaded and overburdened with innumerable information at a very tender age. We are largely deprived of spending time with family or extended families, less verbal and physical interactions with people for developing intellectual capacities. Looking for positive messages through digitalized platforms rather inculcating positive mind set within. This possible chaos and overexposure to the unwanted medium bringing children at the verge of developmental disorders, anxiety, depression, aggression, addiction, bullying and so on. There needs to be a collective and multi-disciplinary approach to regulate the social, mental, physical and spiritual cognition to overcome the psychosomatic disorders. The importance of perspectives and practices for sustainable communities begins with diverse disciplinary interventions and contributions at the early stages of one's life. The importance of evaluation of mental health which impacts communities to sustain needs multi-approach and new methodologies in place to have a greater sustainable impact.

As yoga and meditation are clinically proven methods to deal with mental health disorders, chronic illness and psychosomatic disorders. Naturopathy made its presence felt dealing with cognitive (ADHD, Developmental Disorders) and mental health disorders. Ayurveda and Siddha too have its symptomatic treatments which address mental health and life span perspectives. Wellbeing with a holistic approach has sustainable and life span perspective in the overall development of the resilient communities.

As Yoga is considered a mind-body intervention a healing system and a combination of breathing exercises, physical posture and meditation. According to A.N Singh (2006), Yogic approaches have been successfully used in the management of bronchial asthma, hypertension, ulcer, cervical spondylosis, chronic sinusitis, intractable pain, personality disorder, anxiety, depression, gastritis, rheumatism. Pamela Siegal Nelson Filice(Jan 2015) The incorporation of yoga into the health systems has been encouraged by the WORLD HEALTH ORGANISATION. In Brazil Yoga was included in the Unified Health System (SUS) through bill 719 which created the Academy of Health Program in April 2011. Evidence/ thematic analysis was applied. In Yogic and Complimentary Alternative Medicine system practices, it not only defines the benefit of wellbeing with over all holistic development of sustainable and resilient communities. Multiple interventions can possibly be made with CAM for the lifespan perspectives. CAM, meditation and Yoga has significant scientific evidences in treating sleep disorders, anxiety disorders, schizophrenia and other chronic illness.

\section{Methodology}

Series of the workshop was planned to conduct in three branches of the govt aided schools in Delhi in order to curb, anxiety disorders, teenage depression, addiction and social bullying and extreme aggression. Aggression being the central focus of the workshop where students from 5 standards to 12 standards were asked to attend. There was only one 2 branched which didn't give permission for $10^{\text {th }}$ standard and $12^{\text {th }}$ standard as they are appearing for board exams and the syllabus needs to be covered. Although one branch allowed us to conduct within the shorter time frame and few workshops topics were given permission.

\section{Results}

The study conducted during a series of workshops in a government-aided (DTEA) schools where students have extreme aggression, anxiety, emotional imbalance, performance issues, psychosomatic illness, addictions have drastically decreased at the end of the workshops spanning over six months duration 2 hours twice or thrice weekly on various holistic developmental topics. Students were also taught to perform simple meditation techniques and holistic techniques to cut down the anger and aggression. The students were seen coming from broken families, abusive family backgrounds, fathers mostly drunk and become physically and mentally abusive with their spouse and children. The source of collecting information was based on teacher's remarks and feedback, parents, feedback, principals feedback, and the students have confessed and informed their harmful 
addictions, aggressive behaviour, suicidal tendencies and other related issues by themselves during series of workshops conducted.

The representational graph is taken out to point out larger percentile of students involved majorly in the mentioned indexes.

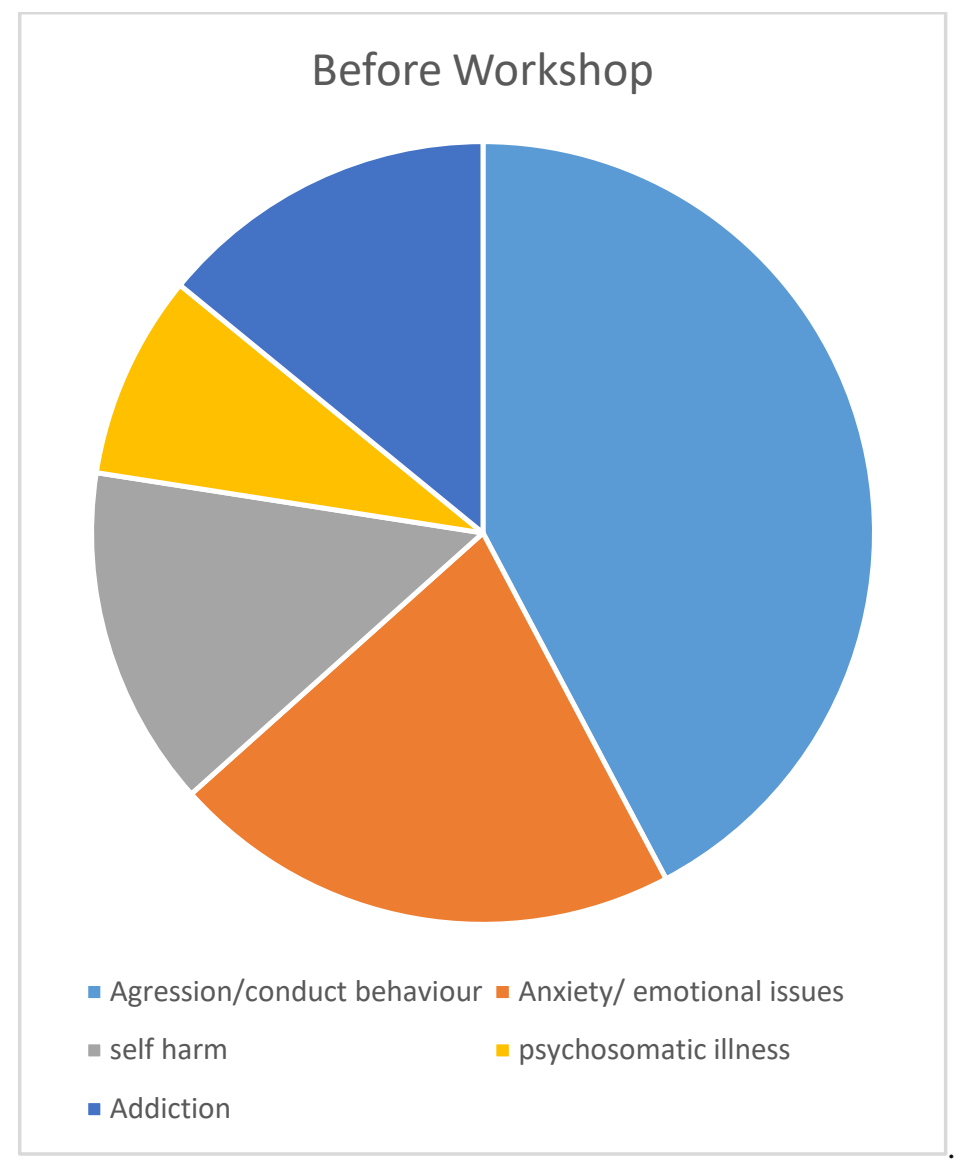




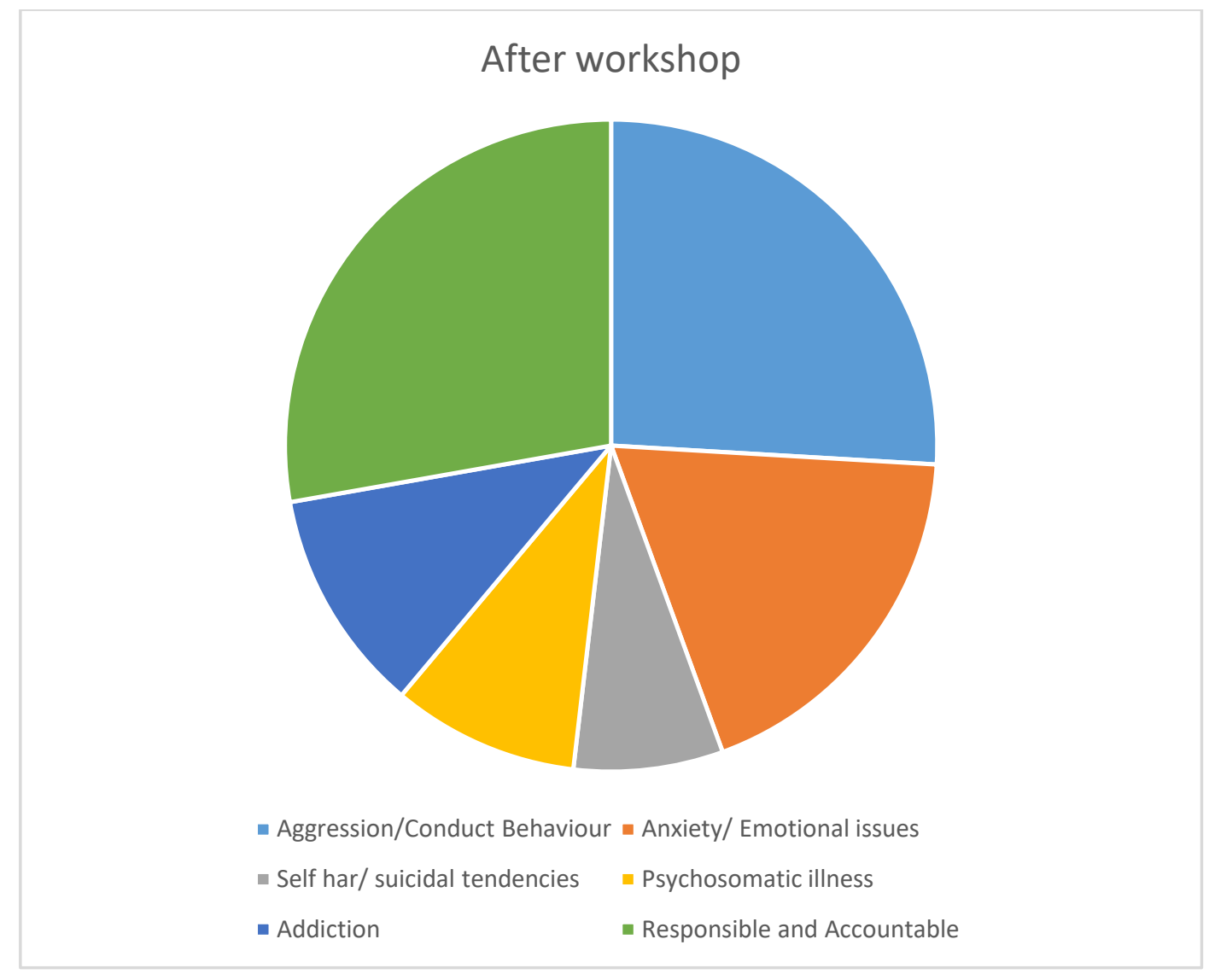

The Feedback was collected from the class and subject teachers, Principal, parents and their peer groups. Teachers remarked that their submission of home work or obeying in the classroom was way better than before and on time. Teachers also felt calling names and using abusive and unparliamentary language also came down a lot. The principal found that their over all obedience in the school discipline and some students have shown visible improvement on conduct behaviour, especially the adolescent students. Teacher and student relationship improved. On the other hand, parents feedback was that there are remarkable behavioural and addictive behaviours that previously shown. The relationship has improved with adjustment, improving on efficacy, decreases anxiety levels with mild depression. The collective feedback is that ( $85 \%)$ students have become responsible for self and others and (58\%) have shown accountability of their actions. The feedback also refers that if such programs continued then there will be remarkable changes in the overall holistic development of the child/ Student.

\section{Conclusion}

The series of workshop based on holistic development of the children, enhancing competencies addressing multiple challenging issues and topics from mental health to skill building for sustaining communities at large. Also suggests that such programs and holistic developmental educative models can be adapted at very stages of life to ensure best practices of wellbeing.

\section{Introduction}

Schizophrenia is a chronic and severe mental disorder that affects how a person thinks, feels, and behaves. Experts believe several factors are generally involved in contributing to the onset of schizophrenia. Evidence suggests that genetic and environmental factor act together to bring about schizophrenia. The condition has an inherited element, but environmental triggers also significantly influence it.Though schizophreniais not as common as other mental disorders, the symptoms can be very disabling. There are several factors that are thought to contribute towards the onset of schizophrenia, like genetic in heritance, a chemical imbalance in the brain, family relationships and environmental factors. The symptoms are classified into four categories: Positive symptoms, Negative symptoms, cognitive symptoms, emotional symptoms. Also the list of major symptoms are Delusions, hallucinations, thought disorders. Other symptoms may include lack of motivation, poor expression of emotions, social withdrawal, unawareness of illness, cognitive difficulties. 
There are no evidence based interventions presently available to manage this disorder and its symptoms. There are evidences although available to treat behavioural, effective thinking, reduced anger, impulsive or distorted thinking through yoga, meditation, walking, and periodical counselling along with dietary modification. However, very few CAM-based treatments and symptomatic interventions are taking place to address psychiatric and neurological disorders. CAM, yoga is extremely encouraging to treat innumerable psychological and behavioural disorders.

\title{
Methodology
}

This case study describes an unplanned trial for a schizophrenic female patient of 51 years old, who have been persistently suffering from the schizophrenic disorder for the past 23 years of her life. She was on medication for more than 10 years and her symptoms sometimes went to impulsive stages of cutting trees, setting the house on fire. Not wearing the right and appropriate attire while being in the company of family or crowd. Extreme physical seizures often witnessed talking and arguing with delusional personality, sudden verbal and physical attacks on the family members and impulsive calmness and remote living from hours to days.

yoga

+meditation

+breathing

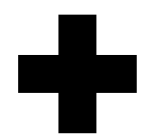

\author{
less frequency of \\ delusions, decreased \\ violent beahviour and \\ became more \\ responsive responsive \\ to the surroundings
}

\section{Diet + Walking+ \\ Counselling}

The patient was on --- medication for over and the time of CAM, yoga, meditation, she was on - and later after 10-15 sessions reduced to ---to pursue the effects of yoga, meditation and counselling, the patient was under continuous observation of her routine and her behaviour patterns. Presently, she is leading a healthy and normal life, no more schizophrenic attacks which were violent in nature. Extremely decreased delusional effects and cognitive imbalance after one month of session. Also, there is a visible stable speech which was unstable and sudden tone modulation to show her extremely violent and aggressive behaviour. Mild aggressive behaviour is seen once in a while after both her sons have got married and she finds it as a threat to her. She is presently on medication and Alternate therapy along with continuous yoga, meditation, walking, and some moderate changes in her diet accordingly.

\section{Conclusion}

This concludes that apart from prescribed medication and continuation of the same if alternative therapy and medicine is practised such as yoga and meditation along with breathing focused exercises, there is evident possibility to control the symptoms of schizophrenic patients.

\section{Introduction}

It is believed clinically that postpartum depression does not have a single cause, but likely results from a combination of physical and emotional factors. After childbirth, hormonal levels ( Estrogen and Progesterone ) in a woman's body quickly descent. This leads to chemical changes in her brain that may trigger mood swings. Mothers are unable to get adequate rest they need to fully recover from giving birth. Sleep deprivation can lead to both physical and mental discomfort and exhaustion, which can contribute to the symptoms of postpartum depression. Clinically effective treatment for postpartum depression is Counselling/ talk therapy and in some cases, medication is suggested. It is believed that if left untreated the postpartum depression can last for months to years. It can affect and interfere with the mother's ability to connect with and care for her newborn baby. 


\section{Methodology.}

The patient had delivered twins (boy and a Girl) immediately resulted in postpartum depression. There was not much family support seen in this case, as she also found that her spouse got engaged in a close friendship with a colleague and she left alone to take care of the kids. The patient is of 28 years old at the time of delivery, working with an IT company. The patient left untreated for longer duration and she attempted self-harming procedures. The patient has also witnessed bouts of mild depression during the third trimester of her pregnancy and there was no support of emotional or physical during this time. Also, she witnessed her spouse being close to the common female friend, which led her to emotional remorse and isolation. When I started my CAM and counselling sessions, it was extremely difficult to gather her attentiveness even for 5 minutes. There were continuous emotional breakdowns and suicidal tendencies. Counselling sessions were intensive lasting up to 45 minutes to an hour. Guided meditation and yognidra were included to calm her down and to be attentive and responsive. There were many episodes of her going back to square one in the initial stages and self -harm was the biggest concern. As her twins were still young ( 7 months $)$ at the time of counselling sessions and timeconsuming chores with the twins. Husband was also given a couple of sessions as he confessed making it difficult for her because of her rude behaviour with the colleague that she mentioned. Intensive, breathing process, yoga nidra and meditation along with counselling for 3 months completely cured her irrational behaviour and postpartum depression. In this case, it was seen that rigorous breathing and meditation practices were done on an everyday basis to stay calm and practice a healthy life style.

\section{Conclusion}

It is also seen that her performance has improved in terms of taking care of her twins and then came a point she was willing to join back to her work happily, though was apprehensive about continuing her work and frequent bouts of depression along with a heavy sigh of crying. Though she conquered all her fears that she developed hypothetically during the postpartum depression, she overcame in 4 months of time with persistent in practising what was told. She has improved the quality of personal life as well as professional life by contributing effective work performance. Her emotional and mental wellbeing graph steadily increased indicating her mindfulness and perseverance. A positive mindset and positive living have been incorporated consciously and today she is the head of her team as a manager at well reputed IT firm. This is the best practices of well being and self-care towards sustainable healthy communities.

\section{Introduction}

Epilepsy is not just one condition, but a group of many different epilepsies with one thing in common is a tendency to have seizures that start in the brain. Epilepsy is usually is diagnosed after a person has had more than one seizure. Not all seizures are due to epilepsy. Your brain comprises of billions of neurons- nerve cells that process and transmit information by interacting with each other. Most neuron interactions occur with few disruptions. Occasionally, small neuron misfires may occur with little consequences. Yet sometimes multiple cells misfire at the same time - depending on the severity and location in the brain. This is a seizure: a sudden, disorganizes electrical discharge in the brain causing muscle twitches and spasms, changes in sensation, mood, behaviour or thought or altered consciousness.

\section{Epilepsy vs Seizures}

A seizure is a single occurrence, whereas epilepsy is a neurological condition characterized by two or more unprovoked seizures.

The patient was aged 21suffering from focal seizures and focal epilepsy since the age of 13 and have been on medication and his condition was getting worse of not being able to handle the frequent seizures along with weak social and communicative skills required for his social participation and further completing his studies. The young boy was isolated by his peers in the college and his seizures made him more depressive and not capable enough to self-care. The boy was treated with counselling in the initial stages and slowly his participation towards breathing techniques have equally increased as part of the schedule. A conscious effort was made to create time to do this as a part of counselling. After 5 sessions, the patient was asked to do certain yoga stretches to begin and later a few other exercises which involved and developed his peripheral vision. After 10 sessions, feedback was taken from his parents, sister and his extended family members. The feedback says that the symptoms have drastically decreased and not a single seizure did take place since the time of the counselling began. He himself has experienced confidence in participating with peers and his classroom participation has improved with his growing graph of performance. The continuous sessions for 15 days and directions given to continue meditation, exercise and breathing techniques have consistently followed. The patient successfully completed his final year and now preparing for Competitive exams. He was also suggested dietary modifications along with the continuous practice of all the alternative therapeutic practices. 


\section{Conclusion}

The patient has now totally dependent on his own and not a single episode of seizure so far and regular followups are made in order to check his routine with meditation and breathing exercise along with dietary modifications. With CAM and yoga and meditation, it is evident that it can be addressed to regularise multiple symptoms and used as a treatment for severe to mild health care systems. The boy has now reduced the medication dosage, yet all other symptomatic issues have been taken care of. The role of CAM and yoga, meditation is larger when we strive for well-being practices and sustainable and resilient communities. It is very evident it can be used for diversified communities with disparities with mindful practices to create a well prepared and positive environment.

\section{References}

[1]. www.researchgate.net/publications/22370114_role_of_yoga_therapies_in_psychosomatic_disorders

[2]. www.jpsychores.com/article/s0022-3999(05)00145-5/fulltext

[3]. www.medicalnewstoday.com/article/36942.php

[4]. www.who.int/news-room/fact-sheet/details/schizophrenia

[5]. www.timesofindia.com/city/bengaluru/13-7-indians-are-mentally-ill-study-says/articleshow/54805096.cms

[6]. www.nimh.nih.gov/health/publications/postpartum-depression-facts/index.shtml\#pub2

[7]. www.hopkinsmedicine.org/neurology_neurosurgery/centers_clinics/epilepsy/conditions.html 